\title{
Meeting report: The 75th Congress of the Japanese Gastric Cancer Association
}

\author{
Yo Kato, Shinichi Nakamura, Kuniyoshi Arai, Toshiro Konishi, Kimiya Takeshita, Takeshi Sano, \\ TAKANORI Hattori, and Mitsuru SASAKo
}

\section{Introduction}

The 75th Congress of the Japanese Gastric Cancer Association was held at the Toshi Center Hotel, Tokyo, February 6-8, 2003, and was attended by 1340 participants. The congress president. Y. Kato of the Cancer Institute of the Japanese Foundation for Cancer Research, adopted the slogan "Moving into the Future through Reflection on the Past" and aimed to organize a meeting where "anything about gastric cancer could be understood."

A total of 530 papers were presented, covering a wide range of gastric cancer topics. Prior to the regular sessions, an educational seminar entitled "Immediate diagnosis and discussion by experts" was held by renowned endoscopists, radiologists, and pathologists, and attracted a large audience.

Discussions about some of the main topics of the congress are summarized below by the chairpersons of each session.

\section{Panel discussion 1. Problems of Group Classification for Gastric Biopsy Specimens (Chaired by T. Aikou, Kagoshima University, and S. Nakamura, Iwate Medical University)}

Group Classifications (GC) for gastric biopsy specimens first appeared in the 8th edition of the General Rules for Gastric Cancer Study in 1971 and were revised in the 11 th edition in 1985. Recently, differences between Japanese and Western pathologists regarding diagnostic criteria in gastrointestinal epithelial neoplasia have become controversial. In September 1998, an interna-

Offprint requests to: Y. Kato, Department of Pathology, Cancer Institute, 1-37-1 Kamiikebukuro, Toshima-ku, Tokyo 170-8455, Japan tional consensus meeting was held in Vienna, where 31 pathologists from 12 countries attempted to reconcile differences in grading and classification. The GC system needed to be reviewed in light of its inherent problems and its inconsistencies with the Vienna Classification (VC). Under these circumstances, a roundtable discussion was held to discuss GC problems.

Three speakers made presentations. H. Watanabe, Niigata University, noted historical aspects of GC and recommended that VC be used instead. R. Schlemper, Fukuoka Medical Clinic, pointed out differences between Japanese and Western pathologists in histological criteria, and stressed the usefulness of the modified VC for histological diagnosis and therapeutic implications. S. Nakamura, Iwate Medical University, reported the results of a questionnaire concerning the problems with GC, to which 152 Japanese pathologists had responded. According to those results, pathologists most routinely used GC, complained about the unclear definition of Group III in GC, and were positive about revising GC to an internationally valid form, but had little interest in $\mathrm{VC}$.

After these presentations, six panelists, A. Ochiai of the National Cancer Center, T. Hamada of the Social Insurance Central General Hospital, T. Yao of Kyushu University, A. Yanagisawa of the Cancer Institute, H. Watanabe, and R. Schlemper discussed the problems regarding GC. T. Yao pointed out inconsistencies in Group III, which includes gastric adenomas and lesions that are difficult to diagnose as either benign (nonneoplastic) or malignant (adenocarcinoma). A. Ochiai cautioned pathologists and clinicians about possible confusion if VC were introduced and used in Japan instead of GC. Category 2 in VC is defined as indefinite for neoplasia, which corresponds to non-neoplastic or neoplastic lesions; and indeed this category might include atypical regenerative epithelium, adenoma, or adenocarcinoma, making it equivalent to Group III in GC. But Category 2 might be misunderstood by 
Japanese pathologists and clinicians as being benign, because they are familiar with the nomenclature of Group II in GC, i.e., as benign. A. Yanagisawa mentioned that Group III in GC is convenient for Japanese pathologists even with its inherently inconsistent definition. T. Hamada, as an endoscopist, stressed that histological diagnostic criteria are a matter of concern for both pathologists and clinicians, stating that every Japanese pathologist needed to have a uniform diagnostic standard for GC. T. Ikou, in closing remarks, noted that through the discussion, the problems with GC might have become more apparent, and that pathologists and clinicians would have to thoroughly discuss whether GC should be revised or VC be introduced instead. (Reported by S. Nakamura.)

\section{Panel discussion 2. Interinstitutional Differences in the Surgical Treatment of Gastric Cancer \\ (Chaired by M. Kitajima, Keio University, Tokyo, and K. Arai, Tokyo Metropolitan Komagome Hospital)}

Interinstitutional differences in surgical treatment may exist in terms of patient prognosis and postoperative complications. Factors causing the differences can be classified into those concerning the patient, the clinician or institution, and the handling of statistical data. In this session, the characteristics of each institution were presented first, then interinstitutional differences and their causes were discussed. An inquiry in both the speakers' and other representative institutions (6 university hospitals and 5 cancer centers) had been conducted in advance, and the results were provided after the discussion.

Concerning patient background, there was no distinct difference in mean age. However, the frequency of patients above 80 years of age varied from $2.8 \%$ to $9.7 \%$. The proportion of patients with early cancer also varied, from $38.4 \%$ to $56.8 \%$.

Among the factors concerning the clinician or the institution, there were no large differences in institutional organization, including the presence of a pathology or chemotherapy division. However, a lack of medical oncologists was indicated by many institutions and was listed as a possible factor in interinstitutional differences.

The annual number of patients differed markedly among institutions (range: 34-288 patients), and the resection rate was also different $(85.0 \%-98.9 \%)$. Although the standard operation was D2 dissection in many institutions, the frequency of D3 dissection reached $25 \%-28 \%$ in some institutions. There were considerable differences among institutions in the 5year survival rate, especially in stage IIIA (48\%-78\%) and IIIB $(17 \%-64 \%)$. The incidence of postoperative complications also differed $(10.6 \%-33.5 \%)$, whereas there were no differences in mortality. There were many comments that the annual number of patients or the number of years of clinical experience showed no effect on therapeutic results.

In the comparison between cancer centers and university hospitals, there were large interinstitutional differences among university hospitals in the degree of lymph node dissection, the distribution of final stage of the disease, and 5-year survival rates. Although the incidence of postoperative complications tended to be high in university hospitals, Y. Kodera, Nagoya University, Aichi, indicated that the frequency of patients with concurrent diseases was also high, and suggested that the differences were not necessarily caused by differences in experience or surgical technique.

In summary, the present reports showed that there were clear interinstitutional differences in surgical treatment and results. However, it was impossible to determine whether those were caused by differences in treatment, patient background, or other factors. As many speakers pointed out, the stage-migration phenomenon due to different treatment policy should always be taken into consideration. We concluded that in order to investigate interinstitutional differences, adjustment of the patient background and standardization of therapeutic methods using the guidelines are necessary. (Reported by K. Arai.)

\section{Panel discussion 3. Contribution of the Clinical Path Concept to the Advancement of Gastric Cancer} Therapy (Chaired by T. Konishi, Kanto Medical Center, NTT East, Tokyo, and Y. Yonemura, Shizuoka Cancer Center)

In the United States the use of clinical pathways (CP) started in 1985, after the introduction of the diagnosisrelated groups/prospective payment system (DRG/ PPS). They were devised mainly to meet the need for improvements in hospital management through such measures as shortening of hospital stay, reducing the cost of hospitalization, and improving the efficiency of nursing. The use of $\mathrm{CP}$ has spread rapidly in Japan recently, where $\mathrm{CP}$ appear to have contributed to an improvement in standardization of treatment and nursing, promotion of team care, and development of risk management. These aspects of $\mathrm{CP}$, rather than the need for improvement of hospital management, seem to have prompted their spread in Japan.

In the area of surgical treatment of gastric cancer, a number of hospitals have started to use CP in the management and care of patients, and this approach is rapidly becoming popular. This panel presented recent advances in $\mathrm{CP}$ on surgical therapy of gastric cancer including laparoscopy-assisted distal gastrectomy 
(LADG), practical application of evidence-based medicine in conjunction with the introduction of $\mathrm{CP}$, the rationale for development of $\mathrm{CP}$, and the revision of $\mathrm{CP}$, finally expanding to development of $\mathrm{CP}$ for electronic medical records.

At the start of the discussion, K. Ezumi, Sakai Municipal Hospital, compared 24 cases treated before the introduction of $\mathrm{CP}$ with an equal number of patients seen thereafter. Significantly improved after CP were the length of hospitalization, medical-fee units claimed from health insurance, duration of nasogastric tube insertion and indwelling urinary catheterization, time to ambulation after operation, number of blood samples drawn, number of days of drain insertion, and number of days of antibiotics administration. It was evident that $\mathrm{CP}$ implementation did not increase complications and was efficacious both in economic and therapeutic terms.

Next, T. Kiyama, Nippon Medical School, Tokyo, compared $44 \mathrm{CP}$-applied cases with 32 conventionally handled cases between January and December 2001. In the $\mathrm{CP}$ cases the overall costs claimed from health insurance (among which were hospital fees, drug costs, and expenses for laboratory tests) decreased, while the perday cost claims increased. Further, because medication and dietary counseling were initiated for many $\mathrm{CP}$ cases, resources were managed effectively by the team, and the costs per capita decreased while medical revenues per bed increased.

K. Shitara, Tokyo Medical and Dental University, indicated that variances were observed in LADG with regard to postoperative period until oral intake, and to the intraoperative manipulation of the cardiac area, but that the CP yielded a favorable postoperative course and enabled a shortening of the hospital stay as well as standardization and streamlining of medical therapy.

M. Inokuchi, Toyama Prefectural Central Hospital, stated that half a year after a CP for distal gastrectomy had been adopted, the first path was revised, and as a result the duration of postoperative infusion therapy as well as that of antibiotics administration and the period of indwelling urinary catheterization decreased gradually; further, the first postoperative bath and the first postoperative rice porridge meal could be taken earlier, and the postoperative hospital stay and the total hospital stay were shortened, thus showing the importance of reevaluation and revision of $\mathrm{CP}$ after initial implementation.

T. Tsujinaka, Osaka National Hospital, reported that introduction of CP fosters standardization and team care. They outlined that in pathways for surgical care for gastric cancer the postoperative hospital stay showed a strong correlation with the schedule for postoperative oral food intake. In contrast to the conventional schedule, a pathway specifying early start of oral intake followed by swift upgrading to rice porridge ad- ministration could be safely implemented, leading to a shortened hospital stay and improvement of nutritional indices. They predicted that in the future the schedule for oral intake after surgery for gastric cancer will come under review throughout Japan.

T. Noie, Kanto Medical Center, NTT East, Tokyo, reported that after use of $\mathrm{CP}$ on electronic medical records (EMR) the postoperative costs decreased from $¥ 417310 \pm 298410$ to $¥ 391940 \pm 23360(P<0.05)$, and reduction of injection costs and examination fees were prominent. $\mathrm{CP}$ on EMR were thought to be of great benefit in helping to reform the current medical system with regard to the management of gastric cancer patients as well as patients with other diseases.

Finally, Chairman T. Konishi introduced the results of a survey on $\mathrm{CP}$ for gastric cancer covering the surgical units of some 1600 Japanese hospitals showing that $74 \%$ of them had adopted a clinical path for one or more surgical procedures, stating that the number of hospitals implementing a path for gastric cancer surgery was increasing gradually year by year. He concluded the panel discussion with the remark that in gastric cancer surgery where variances were exuberant, the application of the clinical path concept will increase patient satisfaction, and it is hoped that in the future the use of $\mathrm{CP}$ will decrease the burden of the medical personnel, while the improvement of the pathways will improve the quality of gastric cancer therapy. (Reported by T. Konishi.)

\section{Panel discussion 4. Review of the Treatment} Guidelines for Gastric Cancer (Chaired by K. Yoshino, Keio University, and K. Takeshita, Tokyo Medical and Dental University)

Two years have passed since the Treatment Guidelines for Gastric Cancer were published by the Japanese Gastric Cancer Association in March 2001. In this session, we focused on the indications and results of endoscopic mucosal resection (EMR) and modified gastrectomies for Stage IA and IB disease, and discussed how the Guidelines, including the manual for general use, are being utilized.

According to the Guidelines, the indications for EMR are mucosal cancers, histologically of differentiated type, without ulceration [UL(-)], and smaller than $2 \mathrm{~cm}$. N. Uedo, Osaka Medical Center for Cancer and Cardiovascular Disease, examined the long-term outcome after EMR for submucosal (SM) cancers and suggested that tumors with SM1 invasion up to $500 \mu \mathrm{m}$ could be treated by EMR alone. E. Nomura, Osaka Medical College, on the other hand, asserted that SM1 lesions larger than $10 \mathrm{~mm}$ may have lymph node metastasis and thus surgical resection should be considered. 
N. Nakagawa, Matsushita Memorial Hospital, Osaka, evaluated the validity of modified gastrectomies for early gastric cancer based on their pathological results of lymph node metastasis, and suggested that some nodal stations should be dissected in addition to the guideline recommendation, e.g., No.14v in antral tumors on the greater curvature.

The use of the Guidelines in daily clinical practice was evaluated by four speakers. S. Shimada, Kumamoto University, found that the Guidelines were used in only $70 \%$ of 22 institutions because the descriptions are sometimes vague and complicated. He presented a flowchart for treatment selection and proposed a major revision of the Guidelines. Other speakers reported the usefulness of having patients read the manual for general use in advance. It helped patients understand the treatment and give informed consent. However, T. Ichikura, National Defense Medical College, said that many patients who had read the manual preferred to be treated according to the experience of the attending surgeon rather than according to the Guidelines.

Following these presentations, M. Sasako of the Guideline committee reported tabulated results of the survey on Guidelines use, current status of reduction surgery, sentinel node biopsy, and laparoscopic gastric surgery.

Finally, the following were pointed out as future issues for the first guidelines in Japan: (1) The treatment prescribed in the manual was carried out according to a combination of clinical paths and guidelines, and this may be associated with surgeons' inattentiveness to their work. (2) There were questions about whether cumulative cases nationwide would show a relation between survival and lymph node dissection based on surgery according to surgical stage. There also were questions about the long-term results of EMR cases according to diagnostic accuracy and methods of EMR. (Reported by K. Takeshita.)

\section{Panel discussion 5. Gastric Cancer in the World (English session) (Chaired by C.A. Rubio, Karolinska Institute, Sweden, and T. Sano, National Cancer Center Hospital, Tokyo)}

According to global estimates in 2000, gastric cancer is the fourth most common cancer, and the second most common cause of cancer death in the world. There is great variation worldwide in the incidence and mortality of the disease, which seems to provide important clues for research on the etiology and prevention of gastric cancer. This international session primarily aimed at giving Japanese researchers and clinicians an occasion to look at the disease from different viewpoints. Although the time and the number of speakers were limited, both chairmen believed that the aim was achieved to a considerable extent.

M. Inoue, National Cancer Center East, Chiba, presented an outline of epidemiological trends of gastric cancer in various regions in the world, and stressed the substantial roles of several environmental factors in etiology of this disease. Helicobacter pylori infection must contribute to the risk of gastric cancer, probably not as a single causative agent but by interaction with other factors including dietary habits. She also highlighted the rapid increase in the West in the rate of cancers at the esophagogastric junction. This appears to be a new disease entity arising in populations with risk factors different from those of classical distal tumors, and close monitoring of this condition is essential.

C.A. Rubio, Karolinska Institute and Hospital, Sweden, examined as a pathologist the gastric cancer specimens in various regions of the world, and found a difference in distribution of gastric intestinal metaplasia (GIM) in the background of carcinoma. He and his colleagues developed a quantitative method for comparing GIM, reviewing 2532 gastrectomy specimens from 7 cities in the Atlantic basin and 6 cities in the Pacific basin, including Tokyo and Matsuyama. Although older patients ( $>60$ years) were included in the Atlantic basin sample, higher scores of GIM were found in the Pacific basin sample. Ethnic differences could not explain the GIM distribution pattern, strongly suggesting the contribution of environmental factors.

Y.I. Kim, Gachon Medical School, Korea, discussed the different histological criteria of gastric carcinoma between WHO and Japanese classifications, especially in well-differentiated adenocarcinoma. While Japanese pathologists make a diagnosis even by cellular atypia alone, those who follow the WHO classification, including most Korean pathologists, diagnose carcinoma only by tumor invasion of the lamina propria. He showed a gradual transition of Korean histologic criteria toward Japanese criteria, and concluded that this will facilitate better understanding of the biological behavior of gastric cancer.

E.K.W. Ng, Prince of Wales Hospital, Hong Kong, was the only surgeon to speak in the session. He said that $H$. pylori did not seem strongly associated with gastric cancer or even intestinal metaplasia in Hong Kong, and he suggested that host genetics, dietary patterns, or both may contribute to carcinogenesis. He showed the results of various clinical trials conducted in Hong Kong and highlighted recent studies of neoadjuvant and topical chemotherapy. (Reported by T. Sano.)

Panel discussion 6. Inflammation and Gastric Cancer (Chaired by N. Kaminishi, University of Tokyo, and T. Hattori, Shiga University of Medical Science) 
Gastric cancer tends to arise in the inflamed stomach. Infection of $H$. pylori and Epstein-Barr virus (EBV), injurious dietary substances, and reflux of bile/ pancreatic juice, as well as other factors, cause inflammatory reactions in the stomach. In this session, possible roles of these various inflammatory factors and host factors were discussed in relation to gastric carcinogenesis.

M. Hatakeyama, Hokkaido University, gave a lecture in which he stated that translocation of H. pylori CagA protein into the gastric epithelial cells dysregulates a cytoplasmic tyrosine phosphatase and leads to increased risk of gastric cancer. This is a new finding related to the molecular mechanism in $H$. pylori carcinogenesis. This is, however, an in vitro study using gastric cancer cell lines. In vivo human studies have not yet been conducted.

A. Sugiyama, Shinshu University, reported the relevance of $H$. pylori gastritis to the development of gastric cancer using an animal model of $H$. pylori-infected Mongolian gerbils and NMU (N-methyl-Nnitrosourea). H. pylori gastritis showed a promoting effect on gastric carcinogenesis. Because a chemical carcinogen was used in the model, the initiating effect of $H$. pylori remained unanswered.

M. Baba, Hamamatsu University, reported that gastric cancer patients with certain polymorphisms in the human $I L-1 B$ gene (such as the T/T and C/T genotypes) were more often infected with $H$. pylori, and those with the $\mathrm{C} / \mathrm{C}$ genotype were not infected. This implied that genotyping provides an important tool in defining highrisk individuals for gastric cancers. Such a host factor in carcinogenesis is very important, but only a few reports on it have appeared.

K. Sakuma, University of Tokyo, studied the role of the $I L-1 \beta$ gene in EBV-associated gastric cancer. They suggested that $I L-1 \beta$ gene polymorphisms make it easy for EBV to infect gastric mucosal cells, and that expression of $I L-1 \beta$ may be essential for morphogenesis of this peculiar type of cancer. However, the possible role of EBV in carcinogenesis was not defined.

M. Narikiyo, National Cancer Center Tokyo, reported the infection of Streptococcus anginosus in gastric cancer patients. They found the bacteria in $44 \%$ (15/ 33 cases) of gastric cancers by the PCR method, and the infection was more preferential in cancers of differentiated type than of poorly differentiated type. The role of $S$. anginosus in carcinogenesis is unknown, but the inflammation itself must be a risk factor, as in the case of H. pylori.

K. Fukuhara from Osaka City University examined the status of $H$. pylori infection in patients who had undergone distal subtotal gastrectomy. H. pylori gastritis was found in the remnant stomach of 52 of 97 patients (42 with Billroth 1, 28 with Billroth 2, and 27 with
Roux-en-Y reconstruction), suggesting that $H$. pylori may play a role in remnant stomach carcinogenesis.

K. Nozaki, Aichi Cancer Center, reported the effect of the timing of eradication in the expression of I kappa $\mathrm{B}$ alpha (I $\mathrm{B} \alpha)$ and $I L-1 \beta$ in the stomach of Mongolian gerbils infected with $H$. pylori and treated with NMU. They found that the earlier the eradication began, the less frequently the gastric cancers occurred in gerbils, and the expression of $\mathrm{I} \mathrm{B} \alpha$ and $I L-1 \beta$ attenuated following $H$. pylori eradication, thereby suggesting that carcinogenesis is dependent on the period and severity of the inflammation.

Video symposium 2. Knacks and Devices in Paraaortic Lymph Node Dissection for Gastric Cancer (Chaired by S. Oyama, Cancer Institute Hospital, Tokyo, and M. Sasako, National Cancer Center Hospital, Tokyo)

In the 1980s, paraaortic lymph node dissection (PAD) was frequently carried out for gastric cancer in Japan. The benefit of this procedure has been evaluated in two randomized controlled studies, the results of which are not yet available. Meanwhile, the use of PAD is limited to cases in which it seems essential to achieve R0 resection, especially in combination with neoadjuvant chemotherapy. Four surgeons presented their own techniques, explaining strategies and devices for PAD.

First, M. Sasako, National Cancer Center, Tokyo, presented the principal technique of PAD (classic method). He showed the basic anatomical structures that are landmarks for a safe procedure and that define the borders of PAD. In his technique, paraaortic nodes in the area between the vena cava (right border of PAD) and the left Gerota's fascia (left border of PAD) are dissected nearly en bloc, without preserving lumbar splanchnics which could be recognized clearly at their branching off from sympathetic chains. To the contrary, he emphasized that the bilateral sympathetic chains and celiac ganglions, together with the greater splanchnic nerves, should be preserved, if not involved, to avoid persistent diarrhea. By mobilizing the left kidney with the entire structure wrapped within the anterior and the posterior Gerota's fasciae, complete dissection of paraaortic nodes behind the left renal pedicle was feasible without dissection of the left adrenal gland.

M. Terashima, Fukushima Medical University, presented a technique for PAD that thoroughly preserves the autonomic nerves. He insisted on preservation of a few lumbar splanchnics and the superior hypogastric plexus on both sides, especially for male patients to avoid ejaculatory disfunction. He compared the morbidity after this procedure with that of classic PAD. The incidence of diarrhea was much lower in those who 
underwent this modified technique, and their performance status 6 months after operation was significantly better than for those undergoing classic PAD.

M. Nomura, Yokosuka City Hospital, reported a similar technique preserving these autonomic nerves. In his technique, the greater and lesser splanchnic nerves are taped, and careful dissection of paraaortic nodes above the renal vein is carried out. He also showed the dissection techniques of the area behind the celiac ganglion and reported a low frequency of diarrhea with this technique.

S. Takahashi, Shizuoka Cancer Center, explained the technique of classic PAD. He used the so-called Mercedes incision to obtain a wider view, which makes PAD easier. He presented techniques of PAD in pa- tients who had two left renal arteries. He emphasized the advantage of autologous blood transfusion for PAD.

Discussion after all the video presentations was focused on preservation of the autonomic nerves. All speakers agreed on the importance of preserving the celiac ganglion with the greater splanchnic nerves. Preservation of the lesser splanchnic nerves and the lumbar splanchnics complicates the technique of the PAD, which may increase operation time and the risk of massive bleeding. The preservation of these nerves is useful to avoid ejaculatory disfunction, which is, however, usually solved after 6-10 months in patients who undergo dissection of these nerves. Further study is needed. (Reported by M. Sasako.) 